\title{
LA INTERPRETACIÓN DE LOS CONTRATOS POR ESCRITO EN INGLATERRA*
}

[Interpretation of Written Contracts in England]

\author{
Neil ANDREws** \\ Universidad de Cambridge, Inglaterra
}

\begin{abstract}
RESUMEN
El artículo examina los principales principios aplicables a la interpretación de los contratos escritos en el derecho inglés, desde el punto de vista del derecho vigente y de la doctrina, incluidos los principios de equidad, así como las atribuciones de los tribunales de apelación o de la Corte Suprema frente a un recurso contra un laudo arbitral.
\end{abstract}

\section{Palabras Clave}

Contratos escritos en Inglaterra Interpretación del contrato en Inglaterra.

\section{Abstract}

This article examines the main principles of the interpretation of written contracts in the English law from the applicable law and doctrine stand point, including the principles of equality as well as the powers of the courts of appeal or the supreme court regarding a recourse against an arbitral award.

\section{KEYWORDS}

Written contracts in England - Interpretation of a contract in England.

RECIBIDO el 20 de abril y ACEPTADo el 23 de junio de 2014

* Traducción de Álvaro Pérez Ragone, profesor de Derecho Procesal Civil de la Pontificia Universidad Católica de Valparaíso; y de Rodrigo Silva Goñi, profesor del curso Reforma Procesal Civil de la Facultad de Derecho de la Universidad Adolfo Ibáñez y asistente de Derecho Civil de la Facultad de Derecho de la Universidad de Chile.

** Profesor de Justicia Civil y Derecho Privado en la Universidad de Cambridge y Bencher of Middle Temple. Correo electrónico: nha1000@cam.ac.uk. 


\section{INTRODUCCIÓN ${ }^{* * *}$}

Este tema es fundamental por el enfoque dado desde el derecho inglés, en vivo contraste con el adoptado en otras jurisdicciones ${ }^{1}$. En aquél hay una búsqueda permanente del significado objetivo del texto que figura en el contrato entre las partes en forma escrita. Los tribunales ingleses no permiten a las partes aportar pruebas de su comprensión personal y subjetiva de lo contenido en el contrato. Tampoco se permite que una parte refiera a las tratativas o negociaciones pre-contractuales con el fin de dilucidar los términos del contrato finalmente acordados. Una excepción a ello se da cuando

${ }^{* * *}$ Abreviaturas: $\mathrm{AC}=$ Appeal Cases; EWCA = England and Wales Hight Court; HLC. $=$ House of Lords Cases; $\mathrm{QB}=$ Queen'sd Bench; UKSC $=$ United Kingdom Supreme Court; WLR. = Weekly Law Report.

${ }^{1}$ Literatura general: ANDrews, Neil, Contract Law (Cambridge, Cambridge University Press 2011), parágr. 14.01-14.32 (la misma bibliografía para el examen del proceso civil); Andrews on Civil Processes (Cambridge, 2013), I: Court Proceedings, cap. $15^{\circ}$. Sobre recursos y derecho arbitral en el derecho ingles: Andrews on Civil Processes (Cambridge, 2013), II; LEWISON, K., Interpretation of Contracts (5a edición, London, 2011) (y el primer suplemento de diciembre del 2013); MCMEel, G., The Construction of Contracts: Interpretation, Implication and Rectification ( $2^{\text {a }}$ edición, Oxford, 2011); McMees, The Interplay of Contractual Construction and Civil Justice: Procedures for Accelerated Justice, en European Business Law Review (2011), pp. 437-449; MitCHELL, Catherine Interpretation of Contracts (London, 2007); desde la perspectiva australiana: Carter, J.W., The Construction of Commercial Contracts (Oxford, 2013). Otro ámbito de discusión: Bingham, Lord, A New Thing Under the Sun: The Interpretation of Contract and the ICS Decision, en Edinburgh Law Review, 12 (2008), pp. 374 ss.; Buxton, R., Construction and Rectification After Chartbrook, en Cambridge Law Journal (2010), pp. 253 ss.; Burrows, A., Construction and Rectification, en Burrows, A. - Peel, E. (coordinadores), Contract Terms (Oxford, Oxford Universiyy Press, 2007), pp. 88 s.; Cartwright, J., Interpretation of English Law in Light of the Common Frame of Reference, en Snijders, H. - Vogenauer, S., (coordinadores), Content and Meaning of National Law in the Context of Transnational Law (Munich, 2009); GRABINER, Lord, The Iterative Process of Contractual Interpretation, en Law 2uaterly Review 41 (2012), pp. 128 ss.; Hoffmann, Lord, The Intolerable Wrestle with Words and Meanings, en South Africa Law Journal (1997), pp. 656 ss.; MCKENDRICK, E., en Worthington, S. (coordinador), Commercial Law and Commercial Practice (Oxford, 2003); McLauchlan, D., Contract Interpretation: What is it About? en Sydney Law Review, 5 (2009), pp. 31 ss.; Nicholls, Lord, My Kingdom for a Horse: the Meaning of Words, en Law Quaterly Review, 121 (2005), pp. 577 ss.; PHiLlips, Lord, The Interpretation of Contracts and Statutes, en Arbitration, 68 (2002), pp. 17 ss.; SpIgelmann C. J., From Text to Contract: Contemporary Contractual Interpretation, en Australian Law Journal, 81 (2007), pp. 322 ss.; Staughton, Sir Christopher, How Do The Courts Interpret Commercial Contracts? en Cambridge Law Journal (1999), pp. 303 ss. 
una de las partes busca una solución equitativa de rectificación del contrato. Dicho recurso se considera en la sección III de este artículo. En esencia, la rectificación es un remedio equitativo que permite al juez insertar nuevas palabras para reflejar el verdadero consenso, lo más objetivamente comprobado, como aquel que se consideró inmediatamente antes de la celebración del contrato ${ }^{2}$ : mediante el remedio de la rectificación se permite al tribunal que declare cuáles términos escritos deben modificarse. Esto siempre que se demuestre que, en su formulación final en el contrato, las partes no lograron reproducir con precisión el consenso previo ininterrumpido, para que ese consenso se manifieste en forma objetiva y pueda tener una subsistencia independiente que fuera discernible.

Por último, el abogado dedicado al derecho civil encontrará notable que en esta área no haya intervenido el legislador. Todas las normas que rigen la interpretación de los contratos, así como la doctrina de equidad que sustenta la rectificación, son creación jurisprudencial que resulta principalmente del examen en apelación de las decisiones judiciales de primera instancia o de los laudos arbitrales en los que se ha aplicado el Derecho contractual inglés ${ }^{3}$. Este monopolio judicial de un importante ámbito del Derecho contractual ha funcionado bien. En efecto, los tribunales siguen siendo competentes para perfeccionar, y a veces incluso para desarrollar con bastante audacia, los principios rectores de la interpretación. De hecho, esto es considerado como el tema más importante del Derecho contractual inglés, no solo desde una perspectiva práctica, sino también en la dinámica doctrinal.

\section{INTERPRETACIÓN}

a) La revisión de los contratos mediante recursos: la construcción de los contratos escritos es una cuestión de derecho y no de hecho. Si el derecho inglés rige la interpretación de todos los "contratos escritos" (incluidos los documentos electrónicos) ${ }^{4}$ es una cuestión de derecho ${ }^{5}$, mientras que la interpretación de los contratos no totalmente formulados por escrito (ya sea oralmente, o en parte oral y parte) es una "cuestión de hecho". Los tribunales de apelación tienen la facultad de revisar

${ }^{2}$ Andrews, Neil, Contract Law (Cambridge, 2011), parágr. 14.33 a 14.51.

${ }^{3}$ En el caso de referencias arbitrales donde el asiento del tribunal se encuentra dentro de Inglaterra y Gales, la High Court de Londres debe primero dar permiso para apelar sobre un punto del derecho inglés, para acceder a la High Court: Arbitration Act, 1996 (Inglaterra y Gales), sec. 69,2 y sec. 69,3.

${ }^{4}$ Beale, Hugh, Chitty on Contracts (31 ${ }^{a}$ edición, London, 2012), I, pp. 12-048.

${ }^{5}$ Ibíd., pp. 12-46. 
primero los errores de relacionados con el derecho, pero en general remiten a las conclusiones de hecho ${ }^{6}$.

b) Objetividad. El "principio del acuerdo objetivo" se opone a una referencia o comprensión no manifestada, subjetiva y personal de una parte en realación a los términos escritos, tanto en su significado como en sus efectos ${ }^{7}$. Lord Hoffmann, en el caso "Investors Compensation Scheme" (1998), dijo: "La interpretación [de contratos escritos] es la constatación del sentido que el documento trasmite a una persona razonable que tenga todo el conocimiento previo que razonablemente puede haber estado a disposición de las partes en la situación en que se encontraban en el momento de celebrar contrato"8.

c) Contexto. Los tribunales adoptarán un enfoque contextual de la interpretación en lugar de un enfoque estrecho según lo que un "diccionario pueda indicar es el significado": así según la declaración de Lord Hoffmann realizada en la causa "Investors Compensation Scheme Ltd v. West Bromwich Building Society" (1998) (que se remonta a las decisiones en los años 1970$)^{10}$. Los tribunales permiten que las partes se refieran a la posición contractual, expresado ello en formas diversas, tales como "objetivo comercial" de la operación, "génesis", "background", "contexto", su ubicación en el correspondiente "mercado"11 o su "medio" 12 . Hay que destacar, sin embargo, que el "background" no se extiende a las negociaciones precontractuales (en ellas, según se verá más abajo, en el caso de un recurso solicitando la rectificación, existe una excepción en relación a la pruebade las negociaciones precontractuales).

d) Necesidad de la disciplina procesal. Lord Hoffmann en el caso "BCCI" (2001) dijo que los tribunales y los árbitros deben frenar a las partes en sus intentos de aportar una cantidad excesiva de información sobre el mérito del caso ${ }^{13}$. Sin perjuicio de que, en "Procter and Gamble Co

\footnotetext{
${ }^{6}$ Andrews, Neil, Civil Processes (Cambridge, 2013), I.

${ }^{7}$ HLC., 1976, “Reardon Smith Line Limited v. Hansen Tangen”, en WLR. (1976), pp. 989 y 996.

${ }^{8}$ HLC., 1998, en WLR., pp. 896 y 912-3.

${ }^{9}$ McKendrick, Ewan, Commercial Law and Commercial Practice (London, 2003), pp. 139-162.

${ }^{10}$ HLC., 1971, "Prenn v. Simonds", en WLR. (1971), pp. 1.381 y 1.384-6, en el caso Lord Wilberforce trazó el acercamiento "anti-literal” en la jurisprudencia de mediados del siglo XIX.

${ }^{11}$ HLC., 1976, "Reardon Smith Line Limited v. Hansen Tangen”, en WLR., pp. 989, 995-6.

${ }^{12}$ HLC., 1997, “Charter Reinsurance Co Ltd v. Fagan”, en AC., pp. 313, 384, Lord Mustill: "Las palabras deben estar establecidas en el paisaje del instrumento como un

${ }^{13}$ HLC., 2001, en AC., p. 251.
} todo". 
v. Svenska Cellulosa Aktiebolaget SCA" $(2012)^{14}$, la herramienta del "CommonLaw" de medida probatoria preventiva de divulgación o exhibición de documentos ${ }^{15}$, ha sido indicada por Rix LJ como un medio adecuado para poder arribar a una correcta formulación de los documentos.

e) Accesibilidad al material de referencia. El "background" correspondiente debe haber sido accesible a las partes actuales: ello fue destacado en el caso "Sigma" (2009) por Lord Collins ${ }^{16}$. Los antecedentes no deben ser enterrados entre los restos arqueológicos de la transacción original establecida entre diferentes personas o entidades -como cuando un documento estándar fue creado por las partes $\mathrm{Xe} Y \mathrm{Y}$ y hace mucho tiempo- pero la actual controversia se refiere a $\mathrm{A}$ y $\mathrm{B}$, que son extraños al documento original, pero que sin embargo lo han adoptado, junto con muchos cientos o incluso miles de otras partes contratantes en el "mercado" en cuestión.

e) Las negociaciones precontractuales y su interpretación. La regla del derecho inglés - no se sigue en la mayoría de otras jurisdicciones del mundo-dice que, cuando se trata de interpretar los contratos escritos (a diferencia de los contratos verbales o escritos parcialmente), una parte no puede referir-sin previa autorización de su contraparte-a las negociaciones previas de las partes. Las cinco razones fundamentalesde ello son(recogidas

${ }^{14}$ EWCA, 2012, Civ. 1413, p. 38: "En el derecho inglés hemos evitado preguntar qué es lo que las partes han efectivamente pretendido, pensando que la cuestión de la intención del contrato debiera poder derivarse objetivamente de su acuerdo, y que cuando surge la disputa sobre la cuestión de la intención efectiva es posible que se sumerja en lo que es una expresión de deseos. En el derecho civil, los asuntos se miran de manera diferente, con el tribunal en libertad, según yo entiendo las cosas, para observarlo todo con el fin de derivar la actual, que no la imputada, intención de las partes. Aun asi, por diferentes razones, el derecho inglés tiene una tendencia mucho mayor que el derecho civil, nuevamente como yo entiendo las cosas, a dar cabida al descubrimiento de documentos e interrogatorio cruzado, a pesar de que aumentan el costo de la litigación. En materia de interpretación contractual hay una ironía en esta combinación de enfoques. No obstante la mayor inclinación a aplicar como yo lo hago la comprensión actual de la interpretación contractual en el derecho inglés, que se ha abierto cada vez más a las influencias de las consideraciones de matrices de hechos y construcción de intenciones, no soy capaz de crear un acuerdo al que las partes podrian o podrian no haber alcanzado, si pensaron y discutieron el problema que ha surgido".

${ }^{15}$ Andrews, Neil, cit. (n. 7), cap. $11^{\circ}$.

${ }^{16}$ Pero en el caso "Sigma", en UKSC, 2009, p. 2, Lord Collins (con el apoyo de los Lords Mance y Hope) desaprobaron de manera muy amplia la indagación de la información del contexto cuando, como en el caso "Sigma", las partes relevantes de la transacción pudieron no haber estado presente al momento de su celebración, y, en cambio, hubieran sido partes de segunda categoría o receptores remotos del conrtato de otras partes que ha estado circulando en el mercado financiero relevante. 
de los fundamentos esbozados por Briggs J. ${ }^{17}$ en primera instancia en el caso "Chartbrook Ltd v. Persimmon Homes Ltd “(2007), basándose en la famosa conferencia de Lord Nicholls: "Mi reino por un caballo"18, sosteniéndose lo siguiente a tener en cuenta en la interpretación de los contratos escritos: $i$ ) la prevención de "incertidumbre e imprevisibilidad"; $i i)$ el hecho de que a los terceros interesados no se les puede garantizar el acceso a dicha historia de la negociación; iii) las relaciones son muy cambiantes y por ello dicha prueba sería inoficiosa; iv) las impresiones que surjan de un solo lado podrían contaminar la investigación socavando el enfoque objetivo de la interpretación; y $v$ ) "los negociadores sofisticados bien podrían tentarse a dejar un rastro en el papel fruto de documentos egoístas y apenas una posición unilateral"19.

f) Negociaciones previas a la celebración del contrato y rectificación. Esta evidencia es la que se aportó a los efectos de rectificación, un remedio equitativo independiente (véase abajo). Y así, las solicitudes de rectificación, a menudo se presentan junto con un escrito sobre la base de una supuesta "interpretación" ordinaria ${ }^{20}$.

g) Conductas posteriores a la celebración del contrato. Un contrato por escrito no debe interpretarse por referencia al comportamiento observado por las partes después de la formación del contrato ${ }^{21}$. Sin embargo, existen dos excepciones: $i$ ) si se puede demostrar que las partes habían acordado específicamente modificar o dejar sin efecto el contrato ${ }^{22}$; o $i$ ) si la doctrina de los actos propios puede dejar entrever la prueba de que, con posterioridad a la formación del contrato, las partes habían acordado implícitamente sobre cómo los términos escritos debían ser interpretados o modificados ${ }^{23}$.

${ }^{17}$ EWHC, 2007, p. 409, cap. $23^{\circ}$.

${ }^{18}$ Lord Nicholls, My Kingdom for a Horse: the Meaning of Words, en Law Quaterly Review, 121 (2005), p. 577; en esta nota de la decision de la House of Lord's' en el caso "Chartbrook", D. McLaughlan (2010), 126, en Law 2uaterly Review, 126 (2010) pp. 8 y 9-11 rechaza varias de estas justificaciones sugeridas.

${ }^{19}$ EWCA, 2008, “Chartbrook v. Persimmon”, Civ 183; All England Reports, 2 (2008) (Commercial), p. 387, cap. $111^{\circ}$, per Collins L.J.; este argumento es descrito como inconvincente por D. McLaughlan en Law 2uaterly Review, 126 (2010), pp. 8 y 11.

${ }^{20}$ MCMeEL, Geard, en European Business Law Review (2011), pp. 437-449.

${ }^{21}$ HLC, 1970, "Whitworth Street Estates (Manchester) Ltd v. James Miller \& Partners Ltd”, AC 583, 603.

${ }^{22}$ Andrews, Neil, cit. (n. 5)

${ }^{23}$ Para establecer tal doctrina, un argumento implícito debe manifestarse en su patrón de comportamiento e interacción: "Amalgamated Investment \& Property Co Ltd v. Texas Commerce International Bank Ltd” (1982), en QB, 84, 120, CA, per Lord Denning M.R.: 'Entonces aqui tenemos [...] evidencia de conductas subsecuentes que vienen en 
b) Sentido común profesional. Los tribunales deben interpretar instrumentos escritos, incluidos los contratos, de una manera acorde a las "constumbres comerciales", con sensibilidad al "sentido común" de la empresa $^{24}$. Hay muchas posiciones doctrinarias que así lo sustentan, como son:

i) Lord Diplock dijo en el caso "Antaios Cia Naviera SA v. Salen Rederierna AB" (1985)25: "si el detallado análisis semántico y sintáctico de las palabras en un contacto comercial conduce a una conclusión que se burla del sentido común empresarial, se debe por el contrario adecuarlo a éste".

ii) Lord Steyn dijo en "Mannai Investment Co v. Eagle Star Life Assurance" (1997) $)^{26}$ : "Las palabras son [...] interpretadas en la forma en que una persona razonable podria interpretarlas en el ejercicio comercial. $Y$ el nivel de la persona comercial razonable es hostil a las interpretaciones técnicas, como en los énfasis indebidos en sutilezas del lenguaje".

iii) Lord Hope apoyó este enfoque en la Corte Suprema en "Multi-Link Ocio v. North Lanarkshire" $(2010)^{27}$, señalando que esto era consistente con los principios de Lord Hoffmann en el caso "Investors' Compensation Scheme Ltd v. West Bromwich Building Society" $(1998)^{28}$.

iv) El Tribunal Supremo ha confirmado este enfoque en el caso "Cielo Lluvioso" (2011) 29, donde Lord Clarke dijo: "[20] A mi juicio no es necesario concluir que, a menos que el significado más natural de las palabras produzca un resultado tan extremo como para sugerir que era indeseado, de modo que el tribunal debe hacer efectivo pues dicho significado [...] [21] . Si hay dos construcciones posibles, el tribunal está habilitado para preferir la construcción que sea más coherente con el sentido común empresarial y rechazar la otra [...] [40] Dado que el lenguaje de [la estipulación contractual pertinente] puede tener dos sentidos, es apropiado para el tribunal tener en cuenta consideraciones de sentido común comercial en la resolución de la cuestión que una persona razonable habria entendido que las partes han pretendido".

v) Y la Corte de Apelaciones en "Procter and Gamble Co v Svenska Cellulosa Aktiebolaget SCA" (2012) hace hincapié en que el caso "Cielo

nuestra ayuda. Está disponible no con el fin de interpretar el contrato, sino para ver cómo ellos mismos actuaron en él. Bajo el pretexto de la preclusión [por convención] podemos prevenir que cualquiera de las partes se remonta a la interpretación que ellos mismos le dieron".

${ }^{24} \mathrm{HL}, 1985$, “Antaios Cia Naviera SA v. Salen Rederierna AB”, AC 191, 201, per Lord Diplock.

${ }^{25} \mathrm{HL}, 1985$, AC 191, 201.

${ }^{26} \mathrm{Hl}, 1997$, AC 749 (una decisión por mayoría relativa a una notificación de renta);

BAKer, P. V., en Law Quaterly Review, 114 (1998), pp. 55-62.

${ }^{27}$ UKSC, 2010, 47; 2011, 1 All ER 175, en [21].

${ }^{28}$ HL, 1998, en WLR., pp. 896, 913.

${ }^{29}$ UKSC, 2011, “Rainy Sky SA v. Kookmin Bank”, en WLR. pp. 2900. 
Lluvioso" no es una orden de reescribir un contrato para lograr un "resultado más justo" (aun suponiendo que ello pueda así ser percibido). En el caso "Procter and Gamble Moore-Bick LJ" sostiene que cuando no hay ambigüedad, el tribunal debe dar efecto al significado claro del contrato ${ }^{30}$.

i) Interpretación reconstructiva del texto ${ }^{31}$. La Cámara de los Lores en "Chartbrook Ltd v. Persimmon Homes Ltd" (2009) ${ }^{32}$ sostuvo que un juez puede interpretar un contrato por la total refundición de una frase o parte relevante de un contrato por escrito cuando: $i$ ) es obvio que la redacción ha sido incorrecta; y ii) también es obvio, como una cuestión de interpretación objetiva, lo que las partes consideraban como el verdadero significado. Así, tanto los principios de interpretación común y la doctrina de la rectificación pueden tener por efecto el obtener la revisión de un documento. Lo más seguro es que una parte que solicite una decisión judicial favorable en un contrato por escrito en disputa para defender tanto la "construcción"(en el sentido en el que el estilo "reconstructivo" se acaba de

${ }^{30}$ EWCA, 2012, Civ. 1413, en cap. $22^{\circ}$, per Moore-Bick LJ: “[...] el punto de partida deben ser las palabras que las partes han utilizado para expresar su intención y en el caso de un acuerdo cuidadosamente alcanzado de este tipo, el tribunal debe cuidar de no caer en la trampa de volver a escribir el contrato con el fin de producir lo que considera como un significado más razonable. En mi opinión, el Acuerdo, considerado en su conjunto, no es razonablemente capaz de contar con dos significados posibles". Rix L.J. añadió en p. 38: "[...] la mayor inclinación a aplicar como yo lo hago la comprensión actual de la interpretación contractual en el Derecho Inglés, que se ha abierto cada vez más a las influencias de las consideraciones de matrices de hechos y construcción de intenciones, no soy capaz de crear un acuerdo al que las partes podrian o podrian no haber alcanzado, si pensaron $y$ discutieron el problema que ha surgido". En los hechos del caso Procter \& Gamble, el tribunal sostuvo que las partes habían acordado que el precio de una costosa planta estaría en Euros, pero el pago de dichas sumas estaría en libras. Las partes no habían llegado a un tipo fijo de conversión de euros a libras. Después de la formación, el tipo de cambio euro/libra varió de manera desventajosa para el comprador. Pero el comprador no pudo demostrar, ya sea por un proceso de interpretación, de implicación de términos, o rectificación, que hubo un consenso de que los Euros fueran convertidos a libras al tipo de cambio (favorable para el comprador) vigente a la fecha del contrato, a diferencia de las fechas posteriores de entrega. Uno de los documentos comerciales intercambiados por las partes llevaba una anotación fijando un tipo de cambio aplicable a esa fecha. Pero esto no tuvo por intención imponer un tipo de cambio fijo. Simplemente estableció un proceso de cálculo hecho en el lugar en ese preciso momento de las relaciones de las partes. En ausencia de una tipo de cambio fijo, el movimiento de la moneda adverso fue a cargo del comprador, y no era tarea del tribunal salvar a esa parte de este resultado económico.

${ }^{31} \mathrm{HL}, 1998$, Caso "Investors Compensation Scheme”, en WLR., pp. 896, 912-3.

${ }^{32}$ UKHL, 2009, AC 1101; señalado D. McLaughlan, D., en Law Quaterly Review, 126 (2010), pp. 8-14. 
explicar) y la "rectificación" (que se resume a continuación) 33 . Hay muchos ejemplos de los tribunales invocando este estilo de interpretación: "Holding \& Barnes plc v. Hill House Hammond Ltd (No 1)" (2001) "34, "Littman v. Aspen Oil (corretaje) Ltd" (2005)35, "KPMG LLP v. Network Rail Infrastructure Ltd" (2007) ${ }^{36}$, "Springwell Navigation Corporation v. JP Morgan Chase" (2010) $)^{37}$ y "Pink Floyd MusicLtd v. EMI Records Ltd" (2011)

j) Situaciones en las que la re-construciónno es posible. Esta reconstrucción no será posible si:

i) El único problema invocado es que ambas partes han entendido mal el alcance de la materia ["Bashirv. Ali" (2011)] ${ }^{39}$; o

ii) Cuando una cláusula es errónea, pero no contiene una solución interna: la Corte de Apelaciones en "ING Bank NV v. Ros Roca SA" (2011) $)^{40}$ sostuvo que no era posible, en los hechos, aplicar la técnica de "interpretación reconstructiva" para volver a escribir una cláusula relativa a un"cobro adicional" de un banco de inversión. Del mismo modo, la tarea de reconstruir el texto fue declarada imposible en "Fairstate Ltd v. General Enterprise \&

${ }^{33}$ McMeEL, Gerard. cit (n. 21)

${ }^{34}$ EWCA, 2001, Civ 1334.

${ }^{35}$ EWCA, 2005, Civ 1579.

${ }^{36}$ EWCA, 2007, Civ 363.

${ }^{37}$ EWCA, 2010, Civ 1221; Contract Law Cases, 705 (2010), pp. 132-140.

${ }^{38}$ EWCA, 2010, Civ 1429; WLR., 1 (2011), p. 770 (Lord Neuberger M.R. and Laws L.J. sostuvieron que un acuerdo para la explotación de los "registros" de Pink Floyd podría interpretarse como abarcando las grabaciones digitales de dicha banda. Decidir lo contrario iría en contra de la finalidad comercial evidente de la transacción. Sin embargo, Carnwath L.J. disintió, encontrando que no había tal error evidente).

${ }^{39}$ EWCA, 2011, Civ 707; Property and Compensation Report, 12 (2011) 2, cap. 39', per Etherton L.J.: [...] este no es un caso [...] en el cual la redacción usada por las partes, en una construcción, conduce a resultados arbitrarios e irracionales'.

${ }^{40}$ EWCA, 2011, Civ 353; WLR. 1 (2012), p. 472 (pero el tribunal fue capaz de lograr un resultado favorable para el banco mediante el empleo de la doctrina de los actos propios por convención, para tener en cuenta las relaciones post contractuales: véanse las pp. 111-112], per Rix L.J., sobre todo este pasaje en [111]: "[...] la doctrina de los actos propios es una doctrina flexible que puede tener en cuenta... la interacción honesta y responsable de las partes de un negocio hacia un contrato. Donde hay lugar para el desacuerdo sobre el sentido o el efecto de un contrato, pero en que las partes han elegido claramente (o pretendido elegir) su propia comprensión del mismo y se han relacionado entre ellas sobre la base de ese entendimiento, si se comprueba que la mutualidad es un supuesto asumido en común, o en señal de conformidad, o en la confianza de una de las partes en la representación de la otra, la doctrina de los actos propios permite que el tribunal en un caso adecuado, dé efecto a las relaciones objetivamente comprobables y mutuas de las partes entre si". 
Management Ltd" $(2010)^{41}$, donde el juez dijo ${ }^{42}$ : "los defectos en el acuerdo registrado en el formulario de garantía son tan fundamentales y extensos, que no pueden ser saneados en forma suficiente, ya sea por la vía de la construcción intencional, rectificación, o cualquier combinación de estos enfoques."(En este caso la rectificación fracasó porque no hubo un consenso claro previo sobre el efecto y el alcance de la garantía.)

k) El tribunal no debe excederse en sus facultades de interpretación. Los tribunales no deben ilegítimamente reescribir el contrato si su significado es claro y no conduce a un absurdo comercial. Así Lord Mustill advirtió en la "Charter Reinsurance Co Ltd v. Fagan" (1997) que no es legítimo que los tribunales o árbitros puedan "imponer a las palabras un significado que ellas no puedan soportar", ya que esto sería "sustituir la negociación realmente hecha por una que el tribunal cree que podría haber sido hecha de mejor manera" ${ }^{\text {‘3. }}$. En similar sentido, Rix LJ señaló en el caso "ING Bank NV v. Ros Roca SA" (2011) ${ }^{44}$ : "los jueces no deben ver en Chartbrook Ltd v Parsimmon Homes Ltd [2009] AC 1101 un ábrete sésamo para la reconstrucción del contrato, sino una oportunidad de remediar mediante la construcción un claro error de lenguaje que las partes no podrian haber pretendido".

${ }^{41}$ EWHC, 2010, 3072 (QB); All England Report (Commercial), 2 (2011), p. 497; 133 Con LR, 112 (Richard Salter QC, Deputy).

42 "[...] los defectos en el acuerdo registrado en el Formulario de Garantía son tan fundamentales y extensos que no pueden ser subsanados suficientemente, ya sea por la construcción intencional, o por rectificación, o por cualquier combinación de estos enfoques.' Las garantías requieren claridad, ibíd. en cap. 93: "[...] es particularmente importante que la Corte deba exigir claridad en cuanto a todas (y no sólo algunas) de las condiciones materiales de la transacción en casos, como el presente, donde se le pide que use sus poderes de construcción intencional o de rectificación para corregir errores en la redacción de un documento en el que se confia se satisfacen los requisitos de la Ley contra el Fraude 1677. Hacer lo contrario conlleva el riesgo de socavar la protección que la ley tenía por objeto conferir.' En cuanto a los derechos del acreedor en los que, por aplicación de la doctrina de los actos propios (por representación mediante el pago en el documento), se impidió al supuesto garante negar la validez del documento, el juez dijo, ibidem, en cap. $97^{\circ}$ : cuesta ver por qué cualquier signatario de un defectuoso acuerdo de garantía no estaría regido por la doctrina de los actos propios. A este respecto, la posición aqui me parece ser muy similar a la considerada por la Cámara de los Lores en Action strength Limited (t/a Vital Resources) v. International Glass Engineering In. Gl. En. Spa [2003 UKHL 17; [2003] 2 AC 541, en el que la pretensión de la doctrina de los actos propios fue rechazada por unanimidad".

${ }^{43} \mathrm{HL}, 1997$,AC, pp. 313, 388.

${ }^{44}$ EWCA, 2011, Civ. 353, en WLR. 472. 


\section{RECTIFICACIÓN ${ }^{45}$}

a) Las dos razones. Hay dos razones distintas para rectificar los contratos por escrito: $i$ ) la rectificación de la intención común basada en un desajuste entre la versión anterior de la transacción que se manifiesta exteriormente y los términos escritos finalmente acordados por las partes; o $i$ ) cuando existe un error unilateral, donde la parte $B$ no ha indicado a la parte A que los términos escritos de su transacción inminente no concuerdan con la comprensión equivocada que $\mathrm{A}$ tiene sobre el contenido de dicho acuerdo por escrito. Analizaremos estos dos temas a continuación.

b) La intención común de rectificación. Un contrato escrito puede ser rectificado en conformidad con el entendimiento precontractual y común de las partes sobre sus términos, siempre que: $i$ ) haya alguna manifestación externa de ese entendimiento ${ }^{46} ; \mathrm{y} i$ ) la "comprensión" surja y sea permitida por ser determinada e interpretada en base al método objetivo. No es suficiente que las dos partes hayan considerado erróneamente que se trataba del objeto X y así utilizar ello en sus relaciones. Si el contrato por escrito luego registra el objeto como $\mathrm{X}$, no hay margen para la rectificación porque no hay una falta de correspondencia entre su consenso previo hacia el exterior y las condiciones fijadas por escrito ${ }^{47}$. El tribunal no tiene cómo "considerar razonable el pedido en relación con una solicitud de rectificación" 48 .

c) Necesidad de una persistente intención en la rectificación. Si la fase anterior de la negociación involucró que las partes acordaran un conjunto de términos $\mathrm{A}, \mathrm{B}, \mathrm{y} \mathrm{C}$, pero la versión final es un conjunto de términos X, Y, y Z, podría quedar claro que las partes han sustituido los elementos A, B y C por unos nuevos elementos como lo son X, Y y Z. Si es ese el caso, no debiera haber ningún margen para rectificar el contrato y restaurar los términos A, B y C. La simple razón por la cual la modificación se deniega es que las partes han sustituido libremente nuevos términos y están contestes con ellos. Así se desprende que la rectificación será apropiado solamente si ha habido una intención permanente e ininterrumpido en celebrar un contrato sobre la base de los términos A, B y C. En los hechos que acabamos de mencionar el consenso no existe ininterrumpidamente y por lo tanto las condiciones finales deben estar: X, Y y Z. La necesidad de que subsista la

${ }^{45}$ Hodge, David, Rectification: The Modern Law and Practice Governing Claims for Rectification for Mistake (London, 2010).

${ }^{46}$ CA, 1970, 2 QB 86, "Joscelyne v. Nissen".

${ }^{47}$ CA, 1953, 2 QB 450, "Rose (Frederick E) (London) Ltd v. Wm H Pim Junr \& Co Ltd".

${ }^{48}$ Neuberger Judge, Property and Compensation Review, 81 (2000) 404, "Holaw Ltd v. Stockton Estates Ltd”, p. 41. 
intención común en forma inalterada surgió en forma dramática en el caso "Daventry" (2011) ${ }^{49}$, por decisión mayoritaria de la Corte de Apelaciones (Toulson LJ y Lord Neuberger MR; Etherton LJ disidente y Vos J). La sorpresiva decisión de la mayoría entraña un conflicto con el proceso elemental de negociación que acabamos de mencionar ${ }^{50}$. Sería decepcionante si esta decisión problemática logra subsistir. No puede ser correcto que el derecho inglés permita la rectificación cuando, durante las negociaciones, se ha producido una clara ruptura en el patrón del lenguaje contractual pertinente, y la versión preferida de una parte se ha impuesto claramente (aplicación de los principios comunes que rigen las negociaciones secuenciales). Si la otra parte no planteó objeciones a esta nueva cláusula claramente contradictoria o nuevo conjunto de términos, y no hay constatación de trato injusto en esta etapa, el contrato debe proceder en estos términos y tal cual como finalmente se estableció.

d) Rectificación de error unilateral. La regla general es que el tribunal no otorgará rectificación simplemente para reflejar la comprensión

${ }^{49}$ Neuberger Judge, EWCA Civ. 1153, 2011, “Daventry District Council v. Daventry \& District Housing Ltd", en cap. $210^{\circ}$ : “[...] estaba siendo aclarado por DDH... que estaban incluyendo un término cuyo efecto era que DDC pagaría el déficit de pensiones, $y$, de hecho, que esto era coherente con la cláusula 14.10.2, que se habia incluido en el proyecto de contrato casi desde el principio". Señalado P. Davies, en Lloyd's Maritime and Commercial Law Review (2012).

${ }^{50}$ En el caso "Daventry" el District Council (DCC) obtuvo exitosamente la rectificación a pesar de que el oponente, una asociación de vivienda (DDH) había introducido claramente en la segunda fase de negociasiones, una cláusula competitiva que inequívocamente contradijo la versión preferida de DDC, y a la que DDC, por consejo legal, apareció objetivamente adhiriendo mediante la celebración final del contrato por escrito en condiciones preferentes para DDH. Sorpresivamente, la mayoría del Tribunal de Apelación (Toulson L.J. y Lord Neuberger M.R.) revocó a Vos J. En opinion de la mayoría, la versión original del documento, como señala Vos J., asignaba la carga financiera al déficit de pensiones de DDH. Durante estas primeras etapas de la negociación, el negociador principal de $\mathrm{DDH}$ percibió que esta redacción podría no ser del todo favorable a DDC, pero no intervino para asegurar que las partes se centraran específicamente en esta incertidumbre del texto (Toulson L.J., en [p. 78, y Lord Neuberger M.R., en pp. 213-225, este último "no sin dudarlo", p. 227) sostuvo que el cambio posterior, especialmente la inserción de la cláusula 14.10.3, iniciada por DDH (esta cláusula inequívoca coloca la carga financiera sobre DDC), no se señaló de manera suficientemente clara a DDC. Por lo tanto, objetivamente, en opinión de la mayoría, este cambio no se superpone a la versión anterior. La mayoría llegó a esta conclusión a pesar de que esta redacción final contradice claramente la versión anterior y a pesar de que esta versión final estuvo disponible para ser leída por los funcionarios de DDC y sus abogados. Pero, respetuosamente, la decisión de Toulson L. J. y Lord Neuberger M.R. es poco convincente. 
errónea de una parte ${ }^{51}$. Sin embargo, la excepción a esta regla se presenta si la parte $\mathrm{B}$ es consciente de que la parte $\mathrm{A}$ se equivoca sobre el contenido o el significado de los términos escritos. Cuando se aplique esa excepción, la rectificación es pues viable. Para ello, B se considera "conscientes del error" del otro en cualquiera de las siguientes tres situaciones: $i$ ) si tenía conocimiento real; o $i$ ) fue voluntariamente ciego frente a un hecho evidente; o iii) que falló intencionalmente o por imprudencia en preguntar si había sido en realidad un error, en contra de la noción de razonabilidad y honestidad ${ }^{52}$. Aunque se ha dicho que la ley no exige la prueba de la "práctica fuerte"53, parece claro que las tres situaciones importan necesariamente una falta de buena fe, o falta de probidad, por parte de $\mathrm{B}^{54}$. La equidad considera que, en las situaciones i) a iii), si B guarda silencio no puede tomar ventaja de un error, y que el contrato se puede rectificar en favor de la contraparte ${ }^{55}$. Esto se justifica sobre la base del actuar injusto y de mala fe de B, o la aquiescencia reprobable por error de una de las partes. Esta es una fuerte intervención equitativa porque la parte equivocada obtiene una "victoria total": un contrato es la refundición en definitiva para reflejar su comprensión unilateral,

${ }^{51}$ CA, 1975, cap. 133, "Riverlate Properties v. Paul”.

${ }^{52} \mathrm{CA}, 1995$, cap. $259^{\circ}, 281 \mathrm{D}, 292 \mathrm{~F}$, “Commission for New Towns v. Cooper (GB) Limited"; EWCA Civ 77, 2005, "George Wimpey UK Ltd v. VI Construction Ltd"; EWHC 1530 (TCC), 2010, "Traditional Structures Ltd v. H W Construction Ltd".

${ }^{53}$ CA, en WLR. 1 (1981), pp. 505, 515 H, per Buckley L.J., "Thomas Bates Ltd v. Wyndham's (Lingerie) Ltd": "Sin duda creo que en ningún caso en la conducta del demandado deba ser tal que haga inequitativo el que se le permita oponerse a la rectificación del documento. Si esto implica necesariamente un cierto grado de "prácticas deshonestas", que así sea; pero por mi parte creo que la doctrina es aquella que depende más de la equidad de la posición".

${ }^{54}$ EWCA, 2005, Civ. 77; Property and Compemnsation Report DG 5, "George Wimpey UK Ltd v. VI Construction Ltd”, p. 79.

55 “A. Roberts \& Co. Ltd v. Leicestershire CC” (1961), cap. 555, 570, Pennycuick J. (señalado R.E. Megarry), en Law Quaterly Review, 77 (1961), pp. 313-316; The Olympic Pride, en Lloyd's Rep., 2 (1980), p. 67, Mustill J; CA, 1981, “Thomas Bates Ltd v. Wyndham's (Lingerie) Ltd”, en WLR., 1 (1984), p. 505; CA, 1984, “Agip Sp A v. Navigazione Alta Italia Sp A, The Nai Genova and the Nai Superba”, en Lloyd's Rep., 1, pp. 353, 365; CA, 1995, cap. $259^{\circ}$ "Commission for New Towns v. Cooper (GB) Limited” (señalado D. Mossop) Journal of Contract Law, 10 (1996), pp. 259-63; EWCA, 2005, Civ. 77, "George Wimpey UK Ltd v. VI Components Ltd”; Property Planning and Compensation Report, 2 (2005), DG 5; EWHC 1530 (Technol. Construction Court, 2010); "Traditional Structures Ltd v. HW Construction Ltd", en pp. 25-31; D. McLauchlan, The "Drastic" Remedy of Rectificationfor Unilateral Mistake, en Law Quaterly Review, 124 (2008), pp. 608-40 (quien piensa que esta categoría de rectificación ha sido malentendida; aunque un juez de primera instancia no tiene la libertad de reconsiderar esta categoría de rectificación debido a que está limitado por la autoridad del Tribunal de Apelación: caso Traditional Structures, ibíd., pp. 32-33; sobre este punto ANDREws, Neil, cit. (n. 3), parágr. 14.47). 
a pesar de que no había una comprensión compartida ${ }^{56}$ en orden a apoyar esa versión del contrato ${ }^{57}$.

e) Estado residual de la rectificación. La rectificación no necesita ser invocada si el tribunal puede, como una cuestión de "construcción simple” (como se explica en la sección II de este artículo), revisar el documento correspondiente. Esto último es posible si $i$ ) está claro que el texto actual no se adecua al sentido común comercial; y ii) resulta evidente cómo se debe reconstruir el documento ${ }^{58}$. Pero la rectificación es una doctrina de último recurso en este sentido. Esta doctrina se aplica sólo si otras técnicas, tales como la interpretación o la implicación ${ }^{59}$ de los términos del derecho $\operatorname{común}^{60}$, no llegan a brindar una solución ${ }^{61}$ adecuada $^{62}$.

f) "Explosión” de procesos de rectificación. Aunque, como se acaba de mencionar, la rectificación es una doctrina de último ratio, se ha producido una "explosion" de los recursos de rectificación. Esto es atribuible a tres factores: en primer lugar, a la creciente complejidad de los contratos escritos comerciales y otros; en segundo lugar, a la tendencia de trabajo con sucesivos borradores integrados con el el estilo "corte y pega" propio de procesamiento de textos, la extensión creciente, multijurisdiccional, y la

${ }^{56}$ EWHC, cap. $32^{\circ}$ (2007), "Rowallan Group Ltd v. Edgehill Portfolio No 1 Ltd”; NPC 9, 2007, p. 14,per Lightman J.: [...] el remedio de la rectificación del error unilateral es una medida drástica, ya que tiene el resultado de la imposición a la parte demandada... un contrato que no pudo, y no tuvo intención, de hacer".

${ }^{57}$ Hodge, David, cit. (n. 46), parágrafos 4-90 a 4-93.

${ }^{58}$ EWCA, 2002, Civ. 1334, Landlord and Tenant Reports, 103, "Holding \& Barnes plc v. Hill House Hammond Ltd ( $\left.\mathrm{N}^{\circ} 1\right)$ ”; EWCA Civ. 1579, 2005, "Littman v. Aspen Oil (Broking) Ltd"; CA, Lloyd's Rep., 1 (1981), p. 633, "Nittan (UK) Ltd v. Solent Steel Fabrication Ltd".

${ }^{59}$ En términos implícitos y en general: ANDREws, Neil, cit. (n. 3), cap. $13^{\circ}$.

${ }^{60}$ Neuberger Judge, Holaw (470) “Ltd v. Stockton Estates Ltd”, 2000, 81 P \& CR 404, p. 41; en p. 44: si un punto es tan evidente que no hace falta decirlo, el juez señaló que la doctrina apropiada es la de los términos implícito, en lugar de la rectificación equitativa.

${ }^{61}$ McGHeE, John y otros, Snells Principles of Equity (32 edición, London, 2010), cap. $16^{\circ}, 16-002$ : "La rectificación no se decretará si el resultado deseado se puede lograr convenientemente por otros medios: por la utilización del derecho consuetudinario, o por acuerdo entre las partes." Snell, en 16-009, también señala que la 'piedra de toque' para las condiciones implícitas, incluso en el contexto de los contratos por escrito, sigue siendo un asunto que requiere de 'necesidad', según lo señaló Sir Anthony Clarke M.R., en 'Mediterranean Salvage \& Towage Ltd v. Seamar Trading \& Commerce Inc', The Reborn' EWCA Civ. 53 (2009); Lloyd's Rep. 2 (2009), p. 639, p. 18; en este caso y su atractivamente escéptica recepción de la discusión de Lord Hoffmann en "AttorneyGeneral for Belize v. Belize Telecom Ltd” (2009) UKPC 10; (2009) 2 All ER 1127, pp. 16-27, especialmente p. 21, ANDREws, Neil, cit. (n. 3), parágr. 13.15.

${ }^{62}$ Véase McMeEL, Gerard, The Construction of Contracts: Interpretation, Implication and Rectification (Oxford University Press, $2^{\mathrm{a}}$ edición, 2011), caps. $10^{\circ}$ y $11^{\circ}$. 
naturaleza multipartita de los contratos modernos; por último, a la riqueza de registros electrónicos accesibles de negociaciones ${ }^{63}$.

g) Rectificación y prueba. La parte que solicite la rectificación debe satisfacer un alto nivel en el estándar y calidad de la prueba, especialmente cuando ambas partes han sido informadas profesionalmente ${ }^{64}$. La rectificación admite mucha más luz en el proceso de iluminar los rincones oscuros del texto escrito que un proceso de interpretación del derecho común. Al considerar una solicitud de rectificación, el tribunal puede admitir pruebas extrínsecas, es decir, la evidencia de discusión o material documental fuera del texto del acuerdo por escrito. Por lo tanto la rectificación es una excepción a la regla "prueba oral" (esta es una regla inglesa especial que rige los contratos escritos) y que sostiene que la prueba fuera del contrato por escrito no puede ser utilizada por un grupo para variar, complementar o contradecir el contenido de ese documento ${ }^{65}$. Y así, la regla de la prueba oral no restringe el proceso de discernir las intenciones y las negociaciones con el propósito de rectificación de precontractual de las partes.

Tampoco todo "acuerdo" en base a parte de una cláusula es una evidencia externa si se aporta la prueba con la demanda de rectificación de un contrato escrito. Una cláusula entera de "acuerdo" es una estipulación en el contrato principal que indica que las partes están contestes en no excluir de su acuerdo ninguna garantía o garantías anteriores y externas, o promesas. Se ha sugerido en primera instancia que sería inapropiado la cláusula entera o completa de "acuerdo " para excluir esas pruebas en este contexto, ya que la función de dicha cláusula es prohibir el recurso a argumentos orales o garantías escritas remotas por satélite independiente del principal contrato por escrito (promesas colaterales o previas). Por el contrario, la rectificación se invoca para demostrar que el contrato principal no registra con precisión el verdadero consenso de las partes ${ }^{66}$.

${ }^{63}$ Hodge, David, cit. (n. 46), p. vii.

${ }^{64}$ EWHC, 2005, 1093, "James Hay Pension Trustees Ltd v. Hird”, p. 81; EWHC 1291, 2010, "Surgicraft Ltd v. Paradigm Biodevices Inc”, p. 69, per Christopher Pycroft (Deputy High Court Judge); EWHC 1530, 2010, “Traditional Structures Ltd v. HW Construction Ltd", p. 34.

${ }^{65}$ En general sobre esta regla, ANDREws, Neil, cit. (n. 3), parágr. 14.26 ss.

${ }^{66}$ EWHC, 2010, 1291, "Surgicraft Ltd v. Paradigm Biodevices Inc", p. 73, per Christopher Pycroft (Deputy High Court Judge); McGHeE, John y otros, cit. (n. 62), cap. 16 16-008; BEALE, Hugh, cit. (n. 5), 5-112. 


\section{RECURSOS FRENTE A LAS DECISIONES}

\section{SOBRE CUESTIONES DE INTERPRETACIÓN O RECTIFICACIÓN}

Si el derecho inglés es aplicable a la operación en cuestión, la interpretación (total) de los contratos por escrito (incluidos los documentos electrónicos) ${ }^{67}$ es una cuestión de derecho ${ }^{68}$. Esto significa que (si se obtiene la admisión del recurso contra la sentencia o "permiso" como lo exige el derecho inglés) ${ }^{69}$ un tribunal de apelaciones ${ }^{70}$ tendrá la oportunidad de reconsiderar la opinión del tribunal de primera instancia de los efectos del contrato $^{71}$ (o el punto podría ser objeto de recurso ante el Tribunal Superior inglés, a saber, el juzgado de lo Mercantil, de un tribunal de arbitraje ${ }^{72}$, si el Alto Tribunal lo permite $)^{73}$.

Por el contrario, los tribunales de apelación son generalmente reacios a reabrir las conclusiones de los hechos efectuada por los tribunales de primera instancia (aunque el alcance exacto de los recursos contra las cuestiones de hecho se ha convertido en un complejo campo de procedimiento): “[...] el enfoque de un tribunal de apelación dependerá del peso que deb a atribuirse a las conclusiones del juez y éste a su vez tiene frente a la Corte de Apelacion la ventaja de ser usualmente reacio a tolerar que esta interfiera en sus decisiones" ${ }^{\prime 74}$. Esa "ventaja" es por ejemplo el monopolio de primera instancia (en la práctica moderna) al escuchar inmediatamente el testimonio directo.

Por otro lado el Tribunal Superior o las decisiones del tribunal de apelación sobre la interpretación de los contratos por escritos suministra decisiones que son precedentes valiosos en tanto uso de palabras o frases estándares en los documentos comerciales. Estas decisiones serán vinculantes para todos los tribunales inferiores, y árbitros que aplican el derecho inglés.

En cuanto a la solución equitativa de rectificación (véase la sección III), un libro de texto principal señala: "aunque los principios aplicables que sustentan la rectificación son una cuestión de derecho, sean o no de un instrumento en particular debe ser rectificado es una cuestión de hecho, [mientras que]

\footnotetext{
${ }^{67}$ BEALE, Hugh, cit. (n. 5), cap. $12^{\circ}, 12-048$.

${ }^{68}$ Ibíd., cap. $12^{\circ}, 12-046$.

${ }^{69}$ Civil Procedural Rules (= CPR.) 52.3(1); 52.4(2).

${ }^{70}$ Sobre el sistema de apelación en procedimientos ante tribunales ingleses, ANDREWs, Neil, cit. (n. 7), I, cap. $15^{\circ}$.

${ }^{71}$ HL, 1996, WLR., 1, p. 1.026, "AXA Reinsurance (UK) v. Field” (1996).

${ }^{72}$ Sobre apelaciones de sentencias arbitrales en cuestiones de derecho inglés, ibíd.,

${ }^{73}$ Véase la Arbitration Act 1996 (Inglaterra), 69; la posibilidad de conceder la autorización para apelar de la decisión de un árbitro se ve limitada: ibíd., 69(3).

${ }^{74}$ EWCA Civ. 1642, 2002, “Assicurazioni Generali SpA v. Arab Insurance Group”; WLR., 1 (2003), p. 577, per Clarke L. judge, p. 15.
} II, 18.67 ss. 
la construcción correcta de un contrato por escrito en particular es una cuestión de derecho. Así apelaciones relativas a la interpretación son mucho más comunes que los recursos sobre la cuestión de la rectificación"75.

\section{OBSERVACIONES FINALES}

Los tribunales ingleses modernos y los árbitros aplican el derecho inglés y ya no están vinculadas a la redacción literal del contrato por escrito, pero pueden tener en cuenta la intención común de las partes en el contexto de la transacción. Frente a una plurarlidad de significados posibles, es legítimo que los tribunales prefieran aquel que refleje mejor las realidades comerciales del contrato o cláusula contractual pertinente.

Los tribunales y árbitros que aplican los principios sustantivos del derecho ingles, poseen el poder liberal de interpretar un contrato por escrito con el fin de darle un nuevo sentido y siempre es objetivamente claro cuando que ha sido defectuoso lo escrito y siempre también que es obvio su verdadero significado. Esta última condición debe permanecer estricta. El tribunal no debe participar en conjeturas o ser creativo de nueva redacción que no está respaldada por una clara implicación: "esto es lo que realmente pretendíamos y habíamos acordado, aunque el documento final no sea inteligible o refleje exactitud".

El derecho contractual inglés no permite referencia a las negociaciones precontractuales al interpretar un contrato escrito. A esta última proposición hay una gran excepción cuando una de las partes busca la solución equitativa de rectificación.

Los tribunales de apelación pueden revisar la decisión de un tribunal de primera instancia (o de la concesión de un tribunal arbitral que ha aplicado derecho sustantivo inglés) ${ }^{76}$ sobre una cuestión de interpretación si el contrato correspondiente está totalmente plasmado por escrito. Esto se debe a que la interpretación de dicho documento se califica como una cuestión de derecho, a diferencia de uno de los hechos (las conclusiones de hecho, si se convierten en la apreciación del tribunal de primera instancia de las pruebas orales, tienden a no ser controvertidos en la apelación). Sin embargo, las apelaciones civiles, incluso sobre cuestiones de derecho, no están disponibles de forma automática. El apelante debe primero solicitar al tribunal de primera instancia o el tribunal de apelación correspondiente la admisión del

${ }^{75}$ McGHeE, John y otros, cit. (n. 62), cap. 16 16.11.

${ }^{76} \mathrm{En}$ el caso de referencias arbitrales donde el asiento del tribunal se encuentra en Inglaterra y Gales, la High Court de Londres debe primero dar permiso para apelar sobre un punto del derecho inglés, para acceder a la High Court: s 69(2) y s 69(3), Arbitration Act (1996, Inglaterra y Gales). 
recurso o permiso para acudir al tribunal de alzada. Si no se da ese permiso, el tribunal de apelación podría pronunciarse con autoridad sobre el punto de la interpretación ${ }^{77}$. La declaración del tribunal de apelación de la metodología pertinente para la obtención de significado entonces será vinculante para los tribunales inferiores y arbitrales que aplican el derecho inglés. El significado de los términos escritos pertinentes, por lo menos en ese contexto inmediato, también será vinculante. De este modo, los tribunales ingleses han construido un rico acervo de decisiones precedentes relativas a las frases tipo de uso comercial. Estas decisiones ayudan a promover la previsibilidad ${ }^{78}$.

\section{BiBLIOGRAFÍA}

Andrews, Neil, Contract Law (Cambridge, Cambridge University Press, 2011). Andrews, Neil, Andrews on Civil Processes (Intersentia, Cambridge, 2013), I.

BeAle, Hugh, Chitty on Contracts (31 a edición, London, 2012).

BONELL, Michael Joachim, The UNIDROIT Principles and CISG. Sources of Inspiration for English Courts, en Bonell, Michael Joachim, The UNIDROIT Principles in Practice: Case Law and Bibliography on the UNIDROIT Principles of International Commercial Contracts (2 a edición, Ardsley, NY, 2006).

Burrows, A. - Peel, E. (coordinadores), Contract Terms (Oxford, Oxford University Press, 2007).

Hodge, David, Rectification: The Modern Law and Practice Governing Claims for Rectification for Mistake (London, 2010).

MCGHEE, John y otros, Snell s Principles of Equity (32a edición, London, 2010).

MCKendrick, Ewan, Commercial Law and Commercial Practice (London, 2003).

McLauchlan, David, The 'Drastic' Remedy of Rectification for Unilateral Mistake, en Law 2uaterly Review, 124.

McMeEL, Gerard, The Construction of Contracts: Interpretation, Implication and Rectification (2a edición, Oxford University, 2011).

MCMEEL, Gerard, The Interplay of Contractual Construction and Civil Justice: Procedures for Accelerated Justice, en European Business Law Review, 437 (2011),

SMith, Marcus, Rectification of Contracts for Common Mistake Law Quaterly Review, $123(2007)$.

${ }^{77}$ Sobre el sistema del "permiso" para apelar en procedimientos ante tribunales ingleses, ANDREws, Neil, cit. (n. 7), parágr. 15.25 ss.

${ }^{78}$ McMeel, Gerard, The Interplay of Contractual Construction and Civil Justice: Procedures for Accelerated Justice, en European Business Law Review, 437 (2011), 438, en el $\mathrm{N}^{\circ} 6$, recoge declaraciones judiciales a favor de la seguridad en el comercio, sobre todo Lord Bingham en el "The Starsin” (2003), en UKHL 12; AC, 1 (2004) 715, p. 13; y Lord Steyn en “The Jordan II”, UKHL 49 (2004); WLR., 1 (2005), pp. 1363, 1370. 